\title{
Convergence Analysis for a Nonsymmetric Galerkin Method for a Class of Singular Boundary Value Problems in One Space Dimension
}

\author{
By Kenneth Eriksson and Yi-Yong Nie*
}

\begin{abstract}
For the method and problems under consideration we estimate the error in the maximum norm as well as at individual nodal points. In order to obtain full superconvergence at all nodal points we have to introduce local mesh refinements, even though the exact solution is smooth for the given class of problems.
\end{abstract}

1. Introduction. In this paper we continue the analysis of a "nonsymmetric" Galerkin type piecewise polynomial approximation procedure introduced in Eriksson and Thomée [1] for a class of singular boundary value problems in one space dimension. We extend the results of [1] to the case of mildly nonlinear problems with the same type of singularity, and analyze the discretization of the stationary problem more closely with respect to superconvergence properties at the nodal points.

We consider thus two related problems, namely the singular two-point boundary value problem

$$
\begin{aligned}
& L u(x)=-u^{\prime \prime}(x)-\frac{b}{x} u^{\prime}(x)=f(x, u(x)) \text { for } x \in I=(0,1), \\
& u^{\prime}(0)=u(1)=0,
\end{aligned}
$$

and the corresponding time-dependent problem

$$
\begin{aligned}
& u_{t}(x, t)+L u(x, t)=f(x, t, u(x, t)) \text { for } x \in I, t>0, \\
& u_{x}(0, t)=u(1, t)=0 \text { for } t>0, \\
& u(x, 0)=u^{0}(x) \text { for } x \in I,
\end{aligned}
$$

where $b$ is a constant, $b \geqslant 1, u^{\prime}=u_{x}=\partial u / \partial x$, and $u_{t}=\partial u / \partial t$. We shall always assume that these problems are well posed, i.e., that the nonlinearities and the data given by the functions $f$ and $u^{0}$ are such that (1.1) and (1.2) admit unique solutions which are sufficiently smooth for all our purposes. (One can prove that this is the case, e.g., if $f$ is sufficiently smooth and if the derivative of $f$ with respect to $u$ admits a certain upper bound depending on $b$.)

Received November 18, 1985; revised June 3, 1986.

1980 Mathematics Subject Classification (1985 Revision). Primary 65N15, 65N30.

Key words and phrases. Nonlinear, singular, two-point boundary value problem, parabolic, spherical symmetry, piecewise polynomial, Galerkin method, error estimate, super convergence.

* Current address. Shenyang Institute of Computer Technology, Shenyang, Liaoning, People's Republic of China. 
Problems of the form (1.1) and (1.2) arise naturally from spherically symmetric problems in higher dimensions. For example, if $u=u(\mathbf{x})$ is the solution of the Dirichlet problem

$$
\begin{aligned}
-\Delta u & =f(|\mathbf{x}|, u) & & \text { in } B, \\
u & =0 & & \text { on } \partial B,
\end{aligned}
$$

where $\mathbf{x}=\left(x_{1}, \ldots, x_{n}\right)$ and $B=\{\mathbf{x}:|\mathbf{x}|<1\}$ is the unit ball in $R^{n}$, then, under suitable assumptions (cf., e.g., [2]), $u$ depends only on $|\mathbf{x}|$, and introducing polar coordinates with $x=|\mathbf{x}|$ one finds that $u(x)=u(|\mathbf{x}|)$ is the solution of (1.1) with $b=n-1$.

Also, the problem of finding a bounded solution of the problem

$$
\begin{aligned}
-U^{\prime \prime}(y) & =F(y, U(y)) \text { for } y \in(1, \infty), \\
U(1) & =0,
\end{aligned}
$$

reduces to (1.1) by means of the transformation of variable $y=x^{-\alpha}$ for $\alpha>0$, giving $b=1+\alpha$ and $f(x, u)=\alpha^{2} x^{-2-2 \alpha} F\left(x^{-\alpha}, u\right)$.

Note that for a smooth solution of the equation in (1.1) or (1.2) the boundary condition at $x=0$ is automatically satisfied because of the singularity in the equation.

For the approximate solution of (1.1) and (1.2) we consider a family of finite-dimensional spaces $\left\{S_{h}\right\}_{0<h \leqslant 1 / 2}$ with $S_{h}$ consisting of all continuous functions which vanish at $x=1$ and which reduce to polynomials of degree at most $r-1$ on each subinterval $I_{i}=\left(x_{i-1}, x_{i}\right)$ of a partition $0=x_{0}<x_{1}<\cdots<x_{N}=1$ of $I$, where $h=\max _{1 \leqslant i \leqslant N} h_{i}$ and $h_{i}=x_{i}-x_{i-1}$.

Following the arguments in [1] we are led to pose as a discrete analogue of (1.1) the problem of finding $u_{h} \in S_{h}$ such that

$$
B\left(u_{h}, v\right)=\left(x f\left(x, u_{h}\right), v\right) \quad \forall v \in S_{h},
$$

where

$$
B(w, v)=\int_{0}^{1}\left(x w^{\prime} v^{\prime}-(b-1) w^{\prime} v\right) d x
$$

and

$$
(w, v)=\int_{0}^{1} w v d x .
$$

Clearly, (1.3) is motivated by the fact that the equation (1.1), after multiplication by $x v$ and integration by parts, can be written in variational form as

$$
B(u, v)=(x f(x, u), v) \quad \forall v \in W,
$$

where $W \equiv\left\{v \in W_{\infty}^{1}(I): v(1)=0\right\}$.

In the same spirit we pose for the approximation of (1.2) the semidiscrete problem of finding $u_{h}=u_{h}(t) \in S_{h}$ such that

$$
\begin{aligned}
\left(u_{h, t}, v\right)+B\left(u_{h}, v\right) & =\left(x f\left(x, t, u_{h}\right), v\right) \quad \forall v \in S_{h}, t>0, \\
u_{h} & =u_{h}^{0} \text { for } t=0,
\end{aligned}
$$

where $u_{h}^{0} \in S_{h}$ is a suitable approximation of $u^{0}$. 
As for the continuous problems, we shall not dwell on the questions of existence and uniqueness of solutions of the nonlinear problems (1.3) and (1.5) but simply assume that these problems are well posed and admit unique solutions. (Again, one can prove that this is the case under appropriate assumptions on the functions $f$.)

In our analysis we shall first consider the time-dependent problem and show that for a particular choice of discrete initial data $u_{h}^{0}$ we have the maximum norm error estimate (Theorem 1)

$$
\left\|\left(u-u_{h}\right)(t)\right\|_{\infty}=O\left(\left(\log \frac{1}{h}\right) h^{r}\right) .
$$

We shall then use smoothing properties of the time-dependent problems to show that for a general initial data approximation of order $O\left(h^{r}\right)$ we have (Theorem 2)

$$
\left\|\left(u-u_{h}\right)(t)\right\|_{\infty}=O\left(t^{-1 / 2}\left(\log \frac{1}{h}\right) h^{r}\right) .
$$

We shall then turn to the stationary problem and show, under suitable assumptions on $f$ (Theorem 3), that for $r>2$ and $h$ sufficiently small,

$$
\left\|u-u_{h}\right\|_{\infty} \leqslant C h^{r}\left\|u^{(r)}\right\|_{\infty}
$$

where $u^{(r)}$ is the $r$ th derivative of $u$, and the constant $C$ only depends on $b, f$, and $r$. For $r=2$ we derive a logarithmic modification of the same estimate.

So far, our results are extensions to the present situation of results obtained in [1] for the case of linear problems.

We next address the question of superconvergence at the nodal points for the discrete solution of the stationary problem. We first consider the case of a general mesh. It appears then (Theorem 4) that the rate of convergence depends on $b$ as well as on $r$, and that the error bound depends on the particular nodal point under consideration in such a way that the superconvergence disappears as we approach $x=0$. In order to obtain high-order rate of convergence uniformly for all nodal points and independently of $b$, we are led to adopt an appropriate mesh refinement strategy near $x=0$. Using such refined meshes we can show (Theorem 5), in the case of a linear equation, that

$$
\max _{1 \leqslant i \leqslant N}\left|\left(u-u_{h}\right)\left(x_{i}\right)\right|=O\left(h^{2 r-2}\right),
$$

i.e., we obtain the same order of superconvergence in the present situation as for the standard Galerkin method for nonsingular problems.

We have organized the paper as follows: In Section 2 we prove some basic lemmas to be used in our subsequent analysis. In Section 3 we analyze the semidiscrete approximation of the time-dependent problem, whereas the discretization for the stationary problem is analyzed in Sections 4 and 5, where the latter contains the superconvergence considerations. Finally, in Section 6 we present some results from test calculations which will illustrate and support our theoretical results.

In the sequel we shall denote by $P_{m}$ the set of polynomials in $x$ of degree $m$. The usual $L_{p}$-norms on $I=(0,1)$ will be denoted $\|\cdot\|_{p}$, or just $\|\cdot\|$ for $p=2$. For the corresponding norms on a subset $J$ of $I$ we shall write $\|\cdot\|_{p, J}$. Throughout the paper we shall denote by $C$ various positive constants which are independent of $h$ and of any particular nodal point under consideration. Let us also recall that we have defined $W$ to be the set of all functions $v$ for which $v(1)=0$ and $v^{\prime} \in L_{\infty}(I)$. 
2. Preliminaries. Here we state and prove some basic lemmas to be used in our subsequent analysis below.

LEMMA 1. If $v$ vanishes at $x=1$, then

$$
\begin{aligned}
& \|v\|_{\infty} \leqslant\left\|v^{\prime}\right\|_{1}, \\
& \|v\| \leqslant\left\|x^{1 / 2} v^{\prime}\right\|,
\end{aligned}
$$

and if $v \in S_{h}$, then

$$
\left\|v^{\prime}\right\|_{1} \leqslant C\left(\log \frac{1}{h_{1}}\right)^{1 / 2}\left\|x^{1 / 2} v^{\prime}\right\|,
$$

where $C$ only depends on $r$.

Proof. The first two inequalities of the lemma follow at once from the identities

$$
v(x)=\int_{1}^{x} v^{\prime}(s) d s=\int_{1}^{x} s^{-1 / 2} s^{1 / 2} v^{\prime}(s) d s
$$

by means of Hölder's inequality. To obtain the third inequality, we first use the equivalence of the $L_{1}$-norms over $I_{1}=\left(0, x_{1}\right)$ and $\left(x_{1} / 2, x_{1}\right)$ for functions in $S_{h}$, and then apply Hölder's inequality.

Our next lemma gives the necessary information about a certain interpolation procedure.

LEMMA 2. Let $v$ be an appropriately regular function vanishing at $x=1$, let $m$ be an integer such that $1 \leqslant m \leqslant r-1$, and let $\tilde{v} \in S_{h}$ be the interpolant of $v$ determined by

$$
\begin{aligned}
& \left.\tilde{v}\right|_{I_{1}} \in P_{m}, \\
& \tilde{v}\left(x_{j-1}\right)=v\left(x_{j-1}\right), \\
& \tilde{v}\left(x_{j}\right)=v\left(x_{j}\right), \\
& \lim _{\substack{s \rightarrow 0 \\
s<0}} \tilde{v}^{(k)}\left(x_{j}+s\right)=\lim _{\substack{s \rightarrow 0 \\
s<0}} v^{(k)}\left(x_{j}+s\right) \quad \text { for } k=1, \ldots, m-1,
\end{aligned}
$$

$j=1, \ldots, N$.

Then, for $j=1, \ldots, N$ and $1 \leqslant p \leqslant \infty$, we have

$$
\begin{aligned}
& \left\|x(v-\tilde{v})^{\prime}\right\|_{1, I_{j}} \leqslant h_{j}^{m}\left\|x v^{(m+1)}\right\|_{1, I_{i},} \\
& \left\|(v-\tilde{v})^{(k)}\right\|_{p, I_{j}} \leqslant h_{j}^{m}\left\|v^{(m+k)}\right\|_{p, I_{,},}, \quad k=0,1 .
\end{aligned}
$$

Proof. For $x \in I_{j}$ we put

$$
h(x, y)= \begin{cases}-\left(x_{j}-x\right)^{m}\left(y-x_{j-1}\right)^{m} /\left(h_{j}^{m} m !\right) & \text { for } x_{j-1} \leqslant y \leqslant x \\ (y-x)^{m} / m !-\left(x_{j}-x\right)^{m}\left(y-x_{j-1}\right)^{m} /\left(h_{j}^{m} m !\right) & \text { for } x<y \leqslant x_{j},\end{cases}
$$

noting that

$$
\begin{aligned}
& h_{y}^{(k)}\left(x, x_{j-1}\right)=0 \text { for } k=0, \ldots, m-1, \\
& h\left(x, x_{j}\right)=0, \\
& h_{y}^{(m+1)}(x, y)=\delta(x-y),
\end{aligned}
$$


where the subscripts $y$ indicate that the derivatives are taken with respect to the second variable of $h=h(x, y)$, and $\delta$ is the Dirac delta distribution. Hence, using integration by parts and the fact that $\left.\tilde{v}\right|_{I_{j}}$ is a polynomial of degree at most $m$, we have (with a slight abuse of the notation of an integral)

$$
\begin{aligned}
(v-\tilde{v})(x) & =\int_{I_{j}} h_{y}^{(m+1)}(x, y)(v-\tilde{v})(y) d y \\
& =(-1)^{m+1} \int_{I_{j}} h(x, y) v^{(m+1)}(y) d y .
\end{aligned}
$$

Differentiating this identity with respect to $x$, and using the fact that

$$
\max _{y \in I_{j}}\left|h_{x}^{\prime}(x, y) / y\right| \leqslant h_{j}^{m-1} / x
$$

we obtain by obvious arguments,

$$
\begin{aligned}
\left\|x(v-\tilde{v})^{\prime}\right\|_{1, I_{j}} & \leqslant h_{j}\left\|x(v-\tilde{v})^{\prime}\right\|_{\infty, I_{j}} \\
& \leqslant h_{j} \max _{x \in I_{j}}\left(x \max _{y \in I_{j}} \mid h_{x}^{\prime}(x, y) / y \|\right)\left\|x v^{(m+1)}\right\|_{1, I_{j}} \\
& \leqslant h_{j}^{m}\left\|x v^{(m+1)}\right\|_{1, I_{j}},
\end{aligned}
$$

which shows the first desired estimate of the lemma. The proof of the $L_{p}$ estimates is similar.

Our third lemma is a local stability type result.

LEMMA 3. Let $g$ and $h$ be given functions and assume that $\theta \in S_{h}$ satisfies

$$
B(\theta, v)=\left(x g, v^{\prime}\right)+(h, v) \quad \forall v \in S_{h} .
$$

Then

$$
\left\|\boldsymbol{\theta}^{\prime}\right\|_{\infty, \omega_{j}} \leqslant C\left(\|g\|_{\infty, \omega_{j}}+\|h\|_{\infty, \omega_{j}}\right) \quad \text { for } 1 \leqslant j \leqslant N
$$

where $\omega_{j}=\left(0, x_{j}\right)$, and $C$ only depends on $b$ and $r$.

Proof. Following the proof of Lemma 4 in [1] we introduce a basis $\left\{\varphi_{i k}\right\}$ for $S_{h}$ by

$$
\varphi_{i k}(x)= \begin{cases}-h_{i} & \text { for } 0 \leqslant x \leqslant x_{i-1}, \\ (-1)^{k-1} h_{i}\left(\left(x-x_{i}\right) / h_{i}\right)^{k} & \text { for } x_{i-1}<x \leqslant x_{i}, \\ 0 & \text { for } x_{i}<x \leqslant 1,\end{cases}
$$

and then have for some coefficients $\theta_{i k}, i=1, \ldots, N, k=1, \ldots, r-1$,

$$
\theta=\sum_{i k} \theta_{i k} \varphi_{i k}
$$

Since the first derivatives of the basis functions $\varphi_{i k}$ are uniformly bounded and vanish outside $I_{i}$, we have

$$
\left\|\theta^{\prime}\right\|_{\infty, I_{t}} \leqslant C \max _{k}\left|\theta_{i k}\right| \text { for } 1 \leqslant i \leqslant N, C=C(r) .
$$

In the proof of Lemma 4 of [1] it is demonstrated that it is possible to construct another basis $\left\{\psi_{i k}\right\}$ for the test functions such that

$$
B\left(\varphi_{i k}, \psi_{j l}\right)=0 \text { for } j \neq i, 1 \leqslant k, l \leqslant r-1,
$$

such that the matrix $B_{i}$ with elements $B\left(\varphi_{i k}, \psi_{i l}\right)$ (where $k$ is the column index) is 
nonsingular with

$$
\left\|B_{i}^{-1}\right\|_{\infty} \leqslant C x_{i}^{-1} h_{i}^{-1}, \quad C=C(r)
$$

and such that

$$
\left\|x \psi_{i l}^{\prime}\right\|_{1}+\left\|\psi_{i l}\right\|_{1} \leqslant C x_{i} h_{i}, \quad C=C(b, r)
$$

where $\psi_{i l}$ is obtained by modifying $\varphi_{i l}$ on $\left(0, x_{i-1}\right)$ so that, in particular, $\psi_{i l} \equiv 0$ on $\left(x_{i}, 1\right)$.

From the representation of $\theta_{i k}$ :

$$
\sum_{k} B\left(\varphi_{i k}, \psi_{i l}\right) \theta_{i k}=B\left(\theta, \psi_{i l}\right)=\left(x g, \psi_{i l}^{\prime}\right)+\left(h, \psi_{i l}\right), \quad l=1, \ldots, r-1,
$$

we thus obtain

$$
\begin{aligned}
\max _{k}\left|\theta_{i k}\right| & \leqslant\left\|B_{i}^{-1}\right\|_{\infty}\left(\|g\|_{\infty, \omega_{i}} \max _{l}\left\|x \psi_{i l}^{\prime}\right\|_{1, \omega_{i}}+\|h\|_{\infty, \omega_{i}} \max _{l}\left\|\psi_{i l}\right\|_{1, \omega_{l}}\right) \\
& \leqslant C\left(\|g\|_{\infty, \omega_{i}}+\|h\|_{\infty, \omega_{l}}\right),
\end{aligned}
$$

which completes the proof of the lemma.

Finally, we shall need some results for an elliptic projection associated with the bilinear form $B(\cdot, \cdot)$.

Lemma 4. Let $P_{B}$ be the projection onto $S_{h}$ defined for all appropriately regular functions $u$ by

$$
B\left(u-P_{B} u, v\right)=0 \quad \forall v \in S_{h},
$$

and put $\rho=u-P_{B} u$. Assuming that $u(1)=0$ we then have

$$
\begin{array}{rlrl}
\left\|\rho^{\prime}\right\|_{\infty} & \leqslant C h^{r-1}\left\|u^{(r)}\right\|_{\infty}, & \\
\|\rho\|_{\infty} \leqslant C\left(\log \frac{1}{h}\right) h^{r}\left\|u^{(r)}\right\|_{\infty} & \text { for } b=1, \\
\|\rho\|_{\infty} \leqslant C h^{r}\left\|u^{(r)}\right\|_{\infty} & \text { for } b>1,
\end{array}
$$

where the constants $C$ only depend on $b$ and $r$.

Proof. Let $\tilde{u} \in S_{h}$ be the interpolant of $u$ defined as in Lemma 2. Then

$$
\left\|(u-\tilde{u})^{\prime}\right\|_{\infty} \leqslant C h^{r-1}\left\|u^{(r)}\right\|_{\infty} .
$$

Further, using Lemma 3 with $g=(u-\tilde{u})^{\prime}$ and $h=-(b-1)(u-\tilde{u})^{\prime}$, we have

$$
\left\|\left(\tilde{u}-P_{B} u\right)^{\prime}\right\|_{\infty}=\left\|\left(P_{B}(\tilde{u}-u)\right)^{\prime}\right\|_{\infty} \leqslant C\left\|(\tilde{u}-u)^{\prime}\right\|_{\infty},
$$

where $C=C(b, r)$. The estimate for $\rho^{\prime}$ then follows by the triangle inequality.

In order to estimate $\rho$ for $b=1$, we introduce the Green's function

$$
\gamma(x, y)= \begin{cases}-\ln y & \text { for } 0 \leqslant x \leqslant y \\ -\ln x & \text { for } y \leqslant x \leqslant 1\end{cases}
$$

by which we can represent $\rho(x)$ in terms of $\rho^{\prime}$ as

$$
\rho(x)=B(\rho, \gamma(\cdot, x)),
$$

where the dot indicates that we integrate with respect to the first argument of $\gamma$. By the definition of $\rho$, we may subtract from $\gamma(\cdot, x)$ in the second argument of $B(\cdot, \cdot)$ any function $v \in S_{h}$. In particular, taking $x$ to be a nodal point $x_{i}$ and $v$ to be the 
interpolant $\tilde{\gamma}$ of $\gamma=\gamma\left(\cdot, x_{i}\right)$ as in Lemma 2, noting that the interpolation procedure of Lemma 2 is well defined and that the corresponding error estimates hold also in the case of a piecewise smooth function such as $\gamma\left(\cdot, x_{i}\right)$, we obtain

$$
\begin{aligned}
\left|\rho\left(x_{i}\right)\right| & \leqslant\left\|\rho^{\prime}\right\|_{\infty}\left\|x(\gamma-\tilde{\gamma})^{\prime}\right\|_{1} \leqslant\left\|\rho^{\prime}\right\|_{\infty} \sum_{j>i} h_{j}\left\|x \gamma^{\prime \prime}\right\|_{1, I_{j}} \\
& \leqslant\left\|\rho^{\prime}\right\|_{\infty} h \int_{x_{i}}^{1} x^{-1} d x=\left\|\rho^{\prime}\right\|_{\infty} h \ln \frac{1}{x_{i}} .
\end{aligned}
$$

Now assume that the maximum of $|\rho|$ is attained at the point $x$, and let $x_{i}$ be a nodal point such that $x_{i}-2 h \leqslant x \leqslant x_{i}$ and $x_{i} \geqslant h$. Then

$$
\begin{aligned}
\|\rho\|_{\infty} & =|\rho(x)|=\left|\rho\left(x_{i}\right)+\int_{x_{i}}^{x} \rho^{\prime}(s) d s\right| \leqslant\left|\rho\left(x_{i}\right)\right|+2 h\left\|\rho^{\prime}\right\|_{\infty} \\
& \leqslant\left(\ln \frac{1}{h}+2\right) h\left\|\rho^{\prime}\right\|_{\infty} \leqslant C\left(\log \frac{1}{h}\right) h^{r}\left\|u^{(r)}\right\|_{\infty},
\end{aligned}
$$

which is the desired bound for $\rho$ in the case $b=1$.

For $b>1$ we similarly use the Green's function

$$
\gamma(x, y)=\frac{1}{b-1} \begin{cases}\left(y^{-(b-1)}-1\right) x^{b-1} & \text { for } 0 \leqslant x \leqslant y \\ \left(1-x^{b-1}\right) & \text { for } y \leqslant x \leqslant 1\end{cases}
$$

to obtain as above with $\gamma=\gamma\left(\cdot, x_{i}\right)$, and with $I \backslash x_{i}=\left(0, x_{i}\right) \cup\left(x_{i}, 1\right)$,

$$
\begin{aligned}
\left|\rho\left(x_{i}\right)\right| & \leqslant\left\|\rho^{\prime}\right\|_{\infty}\left(\left\|x(\gamma-\tilde{\gamma})^{\prime}\right\|_{1}+(b-1)\|\gamma-\tilde{\gamma}\|_{1}\right) \\
& \leqslant C(b)\left\|\rho^{\prime}\right\|_{\infty} h\left(\left\|x \gamma^{\prime \prime}\right\|_{1, \wedge x_{i}}+\left\|\gamma^{\prime}\right\|_{1}\right) \leqslant C(b) h\left\|\rho^{\prime}\right\|_{\infty},
\end{aligned}
$$

from which the desired estimate for $\|\rho\|_{\infty}$ follows as before.

Remark 1 . The factor $\log (1 / h)$ in the estimate for $\|\rho\|_{\infty}$ in Lemma 4 can in fact be removed for $b=1, r>2$. However, for $r=2$ there is an example showing that the given estimate is best possible (cf. Jespersen [3]).

3. The Time-Dependent Problem. In this section we shall consider the time-dependent problem (1.2) and derive error estimates for the solution of the associated semidiscrete problem (1.5) for different choices of discrete initial data $u_{h}^{0}$.

Writing the continuous time-dependent problem in the same variational form as (1.5) we find at once, by subtracting the two, that the error $e=u-u_{h}$ satisfies the equations

$$
\left(x e_{t}, v\right)+B(e, v)=(x q, v) \quad \forall v \in S_{h}, t>0,
$$

where $q=f(u)-f\left(u_{h}\right)$, and $f(u)$ is a shorthand writing for $f(x, t, u(x, t))$.

Since we already have estimates for $\rho=u-P_{B} u$, we write the error with $\theta=P_{B} u-u_{h}$ as

$$
e=\rho+\theta
$$

and note that for $\theta$ we then have

$$
\left(x \theta_{t}, v\right)+B(\theta, v)=(x q, v)-\left(x \rho_{t}, v\right) \quad \forall v \in S_{h}, t>0 .
$$


In the following lemma we collect some basic estimates for $\theta$.

LEMMA 5. Let $u$ and $u_{h}$ be the solutions of (1.2) and (1.5), respectively, and set $\theta=P_{B} u-u_{h}$. Then for $t \in[0, T]$ we have

$$
\begin{aligned}
& \left\|x^{1 / 2} \theta_{x}\right\|^{2} \leqslant C\left(h^{2 r}+\left\|x^{1 / 2} \theta\right\|^{2}+\left\|x^{1 / 2} \theta_{t}\right\|\left\|x^{1 / 2} \theta\right\|\right), \\
& \left\|x^{1 / 2} \theta(t)\right\|^{2}+\int_{0}^{t} B(\theta, \theta) d t \leqslant C\left(h^{2 r}+\left\|x^{1 / 2} \theta(0)\right\|^{2}\right), \\
& \left\|x^{1 / 2} \theta_{t}(t)\right\|^{2} \leqslant C\left(h^{2 r}+\left\|x^{1 / 2} \theta(0)\right\|^{2}+\left\|x^{1 / 2} \theta_{t}(0)\right\|^{2}\right),
\end{aligned}
$$

where the constants $C$ are independent of $h$, but may depend on $b, f, u, r$ and $T$.

Proof. We first put $v=\theta$ in (3.1) to obtain

$$
\begin{aligned}
\left\|x^{1 / 2} \theta_{x}\right\|^{2} & \leqslant B(\theta, \theta)=(x q, \theta)-\left(x \rho_{t}, \theta\right)-\left(x \theta_{t}, \theta\right) \\
& \leqslant C\left(\left\|x^{1 / 2} \rho\right\|^{2}+\left\|x^{1 / 2} \rho_{t}\right\|^{2}+\left\|x^{1 / 2} \theta\right\|^{2}+\left\|x^{1 / 2} \theta_{t}\right\|\left\|x^{1 / 2} \theta\right\|\right),
\end{aligned}
$$

where we have used the fact that

$$
|q| \leqslant C|e| \leqslant C(|\rho|+|\theta|),
$$

since $f$ is smooth. The estimate (i) then follows in view of Lemma 4, since $\rho_{t}=\left(u-P_{B} u\right)_{t}=u_{t}-P_{B} u_{t}$.

With the same choice of $v$ in (3.1) we also have

$$
\frac{1}{2} \frac{d}{d t}\left\|x^{1 / 2} \theta\right\|^{2}+B(\theta, \theta)=(x q, \theta)-\left(x \rho_{t}, \theta\right) \leqslant C\left(h^{2 r}+\left\|x^{1 / 2} \theta\right\|^{2}\right) .
$$

Hence (ii) follows after integration, by Gronwall's lemma.

For the proof of (iii) we differentiate (3.1) with respect to $t$ and then put $v=\theta_{t}$ to obtain

$$
\left(x \theta_{t t}, \theta_{t}\right)+B\left(\theta_{t}, \theta_{t}\right)=\left(x q_{t}, \theta_{t}\right)-\left(x \rho_{t t}, \theta_{t}\right)
$$

or

$$
\frac{1}{2} \frac{d}{d t}\left\|x^{1 / 2} \theta_{t}\right\|^{2}+B\left(\theta_{t}, \theta_{t}\right) \leqslant\left\|x^{1 / 2} q_{t}\right\|^{2}+\left\|x^{1 / 2} \rho_{t t}\right\|^{2}+\left\|x^{1 / 2} \theta_{t}\right\|^{2}
$$

Here,

$$
\begin{aligned}
q_{t} & =f_{t}(u)-f_{t}\left(u_{h}\right)+f_{u}(u) u_{t}-f_{u}\left(u_{h}\right) u_{h, t} \\
& =f_{t}(u)-f_{t}\left(u_{h}\right)+\left(f_{u}(u)-f_{u}\left(u_{h}\right)\right) u_{t}+f_{u}\left(u_{h}\right)\left(u_{t}-u_{h, t}\right)
\end{aligned}
$$

so that

$$
\begin{aligned}
\left\|x^{1 / 2} q_{t}\right\|^{2} & \leqslant C\left(\left\|x^{1 / 2} e\right\|+\left\|x^{1 / 2} e_{t}\right\|\right)^{2} \\
& \leqslant C\left(h^{2 r}+\left\|x^{1 / 2} \theta\right\|^{2}+\left\|x^{1 / 2} \theta_{t}\right\|^{2}\right) .
\end{aligned}
$$

Using also (ii) and the fact that $\left\|x^{1 / 2} \rho_{t t}\right\| \leqslant C h^{r}$, we may thus conclude

$$
\frac{1}{2} \frac{d}{d t}\left\|x^{1 / 2} \theta_{t}\right\|^{2}+B\left(\theta_{t}, \theta_{t}\right) \leqslant C\left(h^{2 r}+\left\|x^{1 / 2} \theta(0)\right\|^{2}+\left\|x^{1 / 2} \theta_{t}\right\|^{2}\right),
$$

from which (iii) follows after integration, using again Gronwall's lemma. This completes the proof of Lemma 5.

We can now prove an error estimate for a particular choice of discrete initial data. 
THEOREM 1. Let $u$ be the solution of (1.2) and $u_{h}$ that of (1.5) with $u_{h}^{0}=P_{B} u^{0}$. Then for $t \in[0, T]$ we have

$$
\|e(t)\|_{\infty} \leqslant \begin{cases}C^{r} \log \frac{1}{h_{1}} & \text { for } b=1, \\ C h^{r}\left(\log \frac{1}{h_{1}}\right)^{1 / 2} & \text { for } b>1,\end{cases}
$$

where $C$ only depends on $b, f, u, r$ and $T$.

Proof. In view of Lemma 4 it suffices to estimate $\theta=P_{B} u-u_{h}$.

By (i) and (iii) of Lemma 1 we have

$$
\|\theta\|_{\infty} \leqslant C\left(\log \frac{1}{h_{1}}\right)^{1 / 2}\left\|x^{1 / 2} \theta_{x}\right\| .
$$

From (3.1) with $v=\theta_{t}$ we have

$$
\begin{gathered}
\left\|x^{1 / 2} \theta_{t}\right\|^{2}+B\left(\theta, \theta_{t}\right)=\left(x q, \theta_{t}\right)-\left(x \rho_{t}, \theta_{t}\right) \\
\leqslant C\left(h^{2 r}+\left\|x^{1 / 2} \theta\right\|^{2}\right)+\frac{1}{2}\left\|x^{1 / 2} \theta_{t}\right\|^{2} .
\end{gathered}
$$

For $t=0$ we have by our choice of initial data that $\theta(0)=0$ and hence, by (3.4), that

$$
\left\|x^{1 / 2} \theta_{t}(0)\right\|^{2} \leqslant C h^{2 r} .
$$

The desired estimate for $\theta$ thus follows from (3.3) and Lemma 5.

We shall now consider the more general situation of arbitrary discrete initial data of appropriate accuracy. For this we have

THEOREM 2. Let $u$ be the solution of (1.2) and $u_{h}$ that of (1.5) with initial data $u_{h}^{0}$ such that

$$
\left\|x^{1 / 2}\left(u^{0}-u_{h}^{0}\right)\right\| \leqslant C_{1} h^{r}
$$

Then for $t \in[0, T]$ we have

$$
\left\|x^{1 / 2} e(t)\right\| \leqslant C h^{r}
$$

and

$$
\|e(t)\|_{\infty} \leqslant C t^{-1 / 2}\left(\log \frac{1}{h_{1}}\right) h^{r},
$$

where the constant $C$ only depends on $b, f, u, C_{1}, r$ and $T$.

Proof. Again, in view of Lemma 4 it suffices to estimate $\theta=P_{B} u-u_{h}$.

By our choice of initial data we have

$$
\left\|x^{1 / 2} \theta(0)\right\| \leqslant\left\|x^{1 / 2} \rho(0)\right\|+\left\|x^{1 / 2}\left(u^{0}-u_{h}^{0}\right)\right\| \leqslant C h^{r} .
$$

The estimate (3.5) thus follows at once by Lemma 5 (ii). 
In order to prove (3.6) we first note by (3.3) and Lemma 5 (i) and (ii) that

$$
\|\theta\|_{\infty}^{2} \leqslant C\left(\log \frac{1}{h_{1}}\right)\left(h^{2 r}+h^{r}\left\|x^{1 / 2} \theta_{t}\right\|\right),
$$

so that it suffices to show that

$$
\left\|x^{1 / 2} \theta_{t}\right\| \leqslant C t^{-1}\left(\log \frac{1}{h_{1}}\right) h^{r}
$$

For this purpose we rewrite (3.2) as

$$
\begin{aligned}
& \frac{1}{2} \frac{d}{d t}\left(t^{2}\left\|x^{1 / 2} \theta_{t}\right\|^{2}\right)+t^{2} B\left(\theta_{t}, \theta_{t}\right) \\
& \quad \leqslant C t^{2}\left(h^{2 r}+\left\|x^{1 / 2} \theta_{t}\right\|^{2}\right)+t\left\|x^{1 / 2} \theta_{t}\right\|^{2} \\
& \quad \leqslant C\left(h^{2 r}+t\left\|x^{1 / 2} \theta_{t}\right\|^{2}\right) .
\end{aligned}
$$

In order to estimate the last term we note from (3.4) that

$$
\left\|x^{1 / 2} \theta_{t}\right\|^{2} \leqslant C h^{2 r}+2\left|B\left(\theta, \theta_{t}\right)\right|
$$

where we also used Lemma 5 (ii). In view of (i) and (iii) of Lemma 1 we have

$$
\begin{aligned}
\left|B\left(\theta, \theta_{t}\right)\right| & \leqslant\left\|x^{1 / 2} \theta_{x}\right\|\left\|x^{1 / 2} \theta_{t x}\right\|+(b-1)\left\|\theta_{x}\right\|_{1}\left\|\theta_{t}\right\|_{\infty} \\
& \leqslant C\left(\log \frac{1}{h_{1}}\right)\left\|x^{1 / 2} \theta_{x}\right\|\left\|x^{1 / 2} \theta_{t x}\right\| \\
& \leqslant C\left(\log \frac{1}{h_{1}}\right) B(\theta, \theta)^{1 / 2} B\left(\theta_{t}, \theta_{t}\right)^{1 / 2} \\
& \leqslant C_{\varepsilon} t^{-1}\left(\log \frac{1}{h_{1}}\right)^{2} B(\theta, \theta)+\varepsilon t B\left(\theta_{t}, \theta_{t}\right),
\end{aligned}
$$

so that with $\varepsilon$ suitably small we conclude from above that

$$
\begin{aligned}
& \frac{1}{2} \frac{d}{d t}\left(t^{2}\left\|x^{1 / 2} \theta_{t}\right\|^{2}\right)+t^{2} B\left(\theta_{t}, \theta_{t}\right) \\
& \quad \leqslant C\left(h^{2 r}+\left(\log \frac{1}{h_{1}}\right)^{2} B(\theta, \theta)\right)+\frac{1}{2} t^{2} B\left(\theta_{t}, \theta_{t}\right) .
\end{aligned}
$$

After integration, using Lemma 5 (ii), we finally obtain that

$$
\begin{aligned}
& t^{2}\left\|x^{1 / 2} \theta_{t}\right\|^{2}+\int_{0}^{t} s^{2} B\left(\theta_{t}, \theta_{t}\right) d s \\
& \leqslant C h^{2 r}+C\left(\log \frac{1}{h_{1}}\right)^{2} \int_{0}^{t} B(\theta, \theta) d s \leqslant C\left(\log \frac{1}{h_{1}}\right)^{2} h^{2 r},
\end{aligned}
$$

which gives the desired estimate (3.7) for $\left\|x^{1 / 2} \theta_{t}\right\|$. This completes the proof of Theorem 2.

4. The Stationary Problem. We now turn our attention to the stationary problem. Assuming that

$$
\lambda=\sup _{\substack{x \in I \\ u}} f_{u}(x, u)<\lambda_{1}
$$


where

$$
\lambda_{1}=\inf _{\substack{v \in W \\ v \neq 0}} \frac{B(v, v)}{(x v, v)}
$$

we shall first show that the maximum norm error in the discrete solution of the stationary problem, determined by (1.3), is of optimal order $O\left(h^{r}\right)$, apart from a logarithmic modification for $b=1$.

We state this in precise terms as

THEOREM 3. Assume that $f$ is appropriately regular and satisfies (4.1), and let $u$ and $u_{h}$ be the solutions of (1.1) and (1.3), respectively. Then, for $h$ sufficiently small and with $e=u-u_{h}$, we have

$$
\|e\|_{\infty} \leqslant C h^{r}\left(\log \frac{1}{h}\right)^{\sigma}\left\|u^{(r)}\right\|_{\infty}
$$

where $C$ only depends on $b, \lambda / \lambda_{1}$, and $\left\|f_{u}\right\|_{\infty}$, and where $\sigma=1$ if $b=1$, and $\sigma=0$ otherwise.

Proof. We shall first show that

$$
\left\|e^{\prime}\right\|_{\infty} \leqslant C h^{r-1}\left\|u^{(r)}\right\|_{\infty}+C\|e\|_{\infty} .
$$

In view of Lemma 4 , this will follow if we show that $\left\|\theta^{\prime}\right\|_{\infty} \leqslant C\|e\|_{\infty}$, where $\theta=P_{B} u-u_{h}$. However, this estimate for $\theta$ follows at once from Lemma 3, since by our variational equations for $u$ and $u_{h}$, and the definition of $P_{B} u$, we have

$$
B(\theta, v)=\left(x\left[f(x, u)-f\left(x, u_{h}\right)\right], v\right)=\left(x f_{u}\left(x, u_{*}\right) e, v\right) \quad \forall v \in S_{h}
$$

for some function $u_{*}$.

We shall now complete the proof by showing that

$$
\|e\|_{\infty} \leqslant C h\left(\log \frac{1}{h}\right)^{\sigma}\left\|e^{\prime}\right\|_{\infty} .
$$

For this we assume that the maximum modulus of $e$ is attained at the point $x$, and take $x_{i}$ to be a nodal point such that $x_{i}-2 h \leqslant x \leqslant x_{i}$ and $x_{i} \geqslant h$. Then, in particular, we have

$$
\|e\|_{\infty}=\left|e\left(x_{i}\right)+\int_{x_{i}}^{x} e^{\prime}(s) d s\right| \leqslant\left|e\left(x_{i}\right)\right|+2 h\left\|e^{\prime}\right\|_{\infty} .
$$

In order to estimate $\left|e\left(x_{i}\right)\right|$, we let $g=g_{i}$ be the solution of the linearized dual problem of (1.3), namely

$$
B(v, g)-(x p v, g)=v\left(x_{i}\right) \quad \forall v \in W,
$$

where $p(x)=f_{u}\left(x, u_{*}(x)\right)$ with $u_{*}$ as above. We claim that (4.4) admits a unique solution $g=g_{i}$ such that

$$
\left\|x g^{\prime \prime}\right\|_{1, I \backslash x_{i}}+\left\|g^{\prime}\right\|_{1} \leqslant \begin{cases}C \log \frac{2}{x_{i}} & \text { for } b=1, \\ C & \text { for } b>1,\end{cases}
$$

where $C$ only depends on $b, \lambda / \lambda_{1}$, and $\left\|f_{u}\right\|_{\infty}$. 
Assume for a moment that this has been proved. Then, taking $v=e$ in (4.4), we find that

$$
\begin{aligned}
e\left(x_{i}\right) & =B(e, g)-\left(x\left[f(x, u)-f\left(x, u_{h}\right)\right], g\right) \\
& =B(e, g-v)-\left(x\left[f(x, u)-f\left(x, u_{h}\right)\right], g-v\right) \quad \forall v \in S_{h},
\end{aligned}
$$

where in the last step we have used (1.1) and (1.3). Taking $v=\tilde{g}$, where $\tilde{g}$ is the interpolant of $g$ defined as in Lemma 2, we deduce that

$$
\begin{aligned}
\left|e\left(x_{i}\right)\right| & \leqslant C\left\|e^{\prime}\right\|_{\infty}\left(\left\|x(g-\tilde{g})^{\prime}\right\|_{1}+\|g-\tilde{g}\|_{1}\right) \\
& \leqslant C\left\|e^{\prime}\right\|_{\infty} h\left(\left\|x g^{\prime \prime}\right\|_{1, I \backslash x_{i}}+\left\|g^{\prime}\right\|_{1}\right),
\end{aligned}
$$

so that (4.3) now follows if we take into account (4.5).

To complete the proof we have to verify our assertions about $g$ and, in particular, (4.5). For this we write (4.4) as an integral equation:

$$
g(x)-K g(x)=\gamma\left(x, x_{i}\right), \quad x \in I,
$$

where

$$
K g(x)=\int_{0}^{1} \gamma(x, y) y p(y) g(y) d y
$$

with $\gamma$ defined as in the proof of Lemma 4. The existence of a unique solution $g \in C(\bar{I})$ of (4.7) follows by Fredholm's third alternative, since clearly $K$ is compact, and since the corresponding homogeneous problem, which we may write as

$$
\tilde{B}(v, \varphi)=B(v, \varphi)-(x p v, \varphi)=0 \quad \forall v \in W,
$$

has only the trivial solution since $\tilde{B}(\cdot, \cdot)$ is positive as a consequence of (4.1). To prove the estimate (4.5), we write $g$ as $\gamma+\varphi$ in (4.4) with $\gamma=\gamma\left(\cdot, x_{i}\right)$, and note that then

Taking $v=\varphi$ we find that

$$
\tilde{B}(v, \varphi)=(x p v, \gamma) \quad \forall v \in W .
$$

$$
\left(1-\frac{\lambda}{\lambda_{1}}\right)\left\|x^{1 / 2} \varphi^{\prime}\right\|^{2} \leqslant \tilde{B}(\varphi, \varphi)=(x p \varphi, \gamma) \leqslant C\|\varphi\|,
$$

where $C$ only depends on $b, \lambda / \lambda_{1}$ and $\|p\|_{\infty}$, and hence, in view of (ii) of Lemma 1 , we conclude that $\|\varphi\| \leqslant C$, and consequently $\|g\| \leqslant C$ with a constant $C$ only depending on $b,\|p\|_{\infty}$, and $\lambda / \lambda_{1}$. Differentiating (4.7), we find that

$$
\left|g^{\prime}(x)\right| \leqslant\left|\gamma_{x}^{\prime}\left(x, x_{i}\right)\right|+C\left\|\gamma_{x}^{\prime}(x, \cdot)\right\|\|g\|,
$$

and thus that $\left\|g^{\prime}\right\|_{1}$ is bounded as in (4.5). Finally, noting that $g$ satisfies the differential equation

$$
-x g^{\prime \prime}(x)+(b-2) g^{\prime}(x)-x p(x) g(x)=\delta\left(x-x_{i}\right) \text { for } x \in I
$$

we have that $\left\|x g^{\prime \prime}\right\|_{1, I \backslash x_{i}}$ admits the same bound. This completes the proof of Theorem 3.

Remark 2 . The number $\lambda_{1}$ can be computed relatively easily. In particular, one can show that

$$
\lambda_{1} \geqslant \inf _{\substack{v \in W \\ v \neq 0}} \frac{\left\|x^{1 / 2} v^{\prime}\right\|^{2}}{\left\|x^{1 / 2} v\right\|^{2}}=5.783 \ldots
$$

(The number $5.783 \ldots$ is the square of the smallest root of the Bessel function of order zero.) 
Remark 3. The factor $\log (1 / h)$ for $b=1$ in the estimate of Theorem 3 can be removed for $r>2$ (cf. Remark 1). This will be clear from our subsequent analysis.

5. Superconvergence. We shall now carry out a more precise analysis of the size of the error at the interior nodal points $x_{i}$ for our discretization of the stationary problem, looking for superconvergence, i.e., convergence better than $O\left(h^{r}\right)$, at these points. Our analysis, together with some numerical tests which will be presented below, indicate that the rate of convergence at a given such point is not always of order $O\left(h^{2 r-2}\right)$ in the present situation, even for a smooth solution, but may depend on $b$. Furthermore, it appears that the extra convergence that is obtained at the nodal points in general decreases and disappears as we approach $x=0$. In order to obtain superconvergence of order $O\left(h^{2 r-2}\right)$ independently of $b$ and uniformly at all nodal points $x_{i}$, we shall introduce nonuniform meshes with local refinements near $x=0$.

For simplicity, we shall consider here only the case of linear problems. For a general mesh we can then prove the following

THEOREM 4. Assume that $f(x, y)=p(x) y$ satisfies (4.1), i.e., that $\sup _{x \in I} p(x)<$ $\lambda_{1}$, and that $p$ is appropriately regular, and let $u$ and $u_{h}$ be the solutions of (1.1) and (1.3), respectively. Then, for $h$ sufficiently small we have at the interior nodal points $x_{i}$, for $b=1$,

$$
\left|e\left(x_{i}\right)\right| \leqslant \begin{cases}C h^{4} x_{i}^{-1} & \text { for } r=3 \\ C h^{6}\left(\log \left(1 / x_{i}\right) \log (1 / h)+x_{i}^{-2}\right) & \text { for } r=4 \\ C\left(h^{r+2} \log \left(1 / x_{i}\right)+h^{2 r-2} x_{i}^{2-r}\right) & \text { for } r>4\end{cases}
$$

and for $b>1$,

$$
\left|e\left(x_{i}\right)\right| \leqslant \begin{cases}C h^{2 r-2} x_{i}^{2-r} & \text { for } r<b+1, \\ C h^{2 r-2} \log (1 / h) x_{i}^{2-r} & \text { for } r=b+1, \\ C h^{r+b-1} x_{i}^{1-b} & \text { for } r>b+1,\end{cases}
$$

where the constants $C$ depend on $b, f$, and $u$.

Proof. We recall from the proof of Theorem 3 that

$$
\left|e\left(x_{i}\right)\right| \leqslant C\left\|e^{\prime}\right\|_{\infty}\left(\left\|x(g-\tilde{g})^{\prime}\right\|_{1}+\|g-\tilde{g}\|_{1}\right),
$$

where $g=g_{i}$ is the associated Green's function, and $\tilde{g} \in S_{h}$ its interpolant as in Lemma 2.

As in the proof of Theorem 3, we have

$$
\begin{aligned}
\left\|e^{\prime}\right\|_{\infty} & \leqslant\left\|\rho^{\prime}\right\|_{\infty}+\left\|\theta^{\prime}\right\|_{\infty} \leqslant C h^{r-1}\left\|u^{(r)}\right\|_{\infty}+C\|e\|_{\infty} \\
& \leqslant C h^{r-1}\left\|u^{(r)}\right\|_{\infty} .
\end{aligned}
$$

It now remains to show the appropriate bounds for the interpolation error $g-\tilde{g}$. In order to do this we need to determine the regularity of $g=g_{i}$.

In the proof of Theorem 3 we showed that $\|g\| \leqslant C$ for some constant $C$ independent of $i$. In fact, in view of (4.7), it then follows that

$$
\|g\|_{\infty} \leqslant \begin{cases}C \log \left(2 / x_{i}\right) & \text { for } b=1 \\ C(b) & \text { for } b>1 .\end{cases}
$$


Differentiating (4.7), we find for $b=1$ that

$$
\begin{aligned}
g^{(m)}(x)= & \gamma^{(m)}\left(x, x_{i}\right)+c_{m} x^{-m} \int_{0}^{x} y p(y) g(y) d y \\
& +\sum_{j=1}^{m-1} \sum_{k=0}^{j-1} c_{m j k} x^{-m+j} g^{(k)}(x)[x p(x)]^{(j-k-1)},
\end{aligned}
$$

where the last sum drops out for $m=1$. We therefore have for $b=1$,

$$
\begin{aligned}
\left|g^{(m)}(x)\right| \leqslant & \left|\gamma^{(m)}\left(x, x_{i}\right)\right|+C x^{-m} \int_{0}^{x} y|g(y)| d y \\
& +C \sum_{k=0}^{m-2}\left|g^{(k)}(x)\right| x^{-m+k+2},
\end{aligned}
$$

and consequently, using this inequality iteratively, i.e., to estimate also the quantities $\left|g^{(k)}(x)\right|$ appearing on the right, and then (5.1) and the definition of $\gamma$, we obtain

$$
\begin{aligned}
\left|g^{(m)}(x)\right| \leqslant & \left|\gamma^{(m)}\left(x, x_{i}\right)\right|+C x^{-m} \int_{0}^{x} y|g(y)| d y \\
& +C \sum_{k=0}^{m-2}\left|\gamma^{(k)}\left(x, x_{i}\right)\right| x^{-m+k+2} \\
& \leqslant \begin{cases}C x^{-m+2} \log \left(2 / x_{i}\right) & \text { for } 0<x<x_{i}, \\
C x^{-m} & \text { for } x_{i}<x<1,\end{cases}
\end{aligned}
$$

again with the convention that the sum drops out for $m=1$. Similarly, we have for $b>1$,

$$
\begin{aligned}
g^{(m)}(x)= & \gamma^{(m)}\left(x, x_{i}\right) \\
& +c_{b m} x^{b-1-m}\left(-\int_{0}^{x} y p(y) g(y) d y+\int_{x}^{1} y\left(y^{1-b}-1\right) p(y) g(y) d y\right) \\
& +\sum_{k=0}^{m-2} c_{b m k} g^{(k)}(x) x^{-(m-2)+k},
\end{aligned}
$$

giving first

$$
\begin{aligned}
\left|g^{(m)}(x)\right| \leqslant & \left|\gamma^{(m)}\left(x, x_{i}\right)\right|+C x^{b-1-m} \int_{0}^{x} y|g(y)| d y \\
& +C \int_{x}^{1} y\left(y^{1-b}-1\right)|g(y)| d y+C \sum_{k=0}^{m-2}\left|g^{(k)}(x)\right| x^{-(m-2)+k},
\end{aligned}
$$

and then, by the same arguments as above,

$$
\begin{aligned}
\left|g^{(m)}(x)\right| \leqslant & \left|\gamma^{(m)}\left(x, x_{i}\right)\right|+C \sum_{k=0}^{m-2}\left|\gamma^{(k)}\left(x, x_{i}\right)\right| x^{-(m-2)+k} \\
& +C x^{b-1-m}\left(\int_{0}^{x} y|g(y)| d y+\int_{x}^{1} y\left(y^{1-b}-1\right)|g(y)| d y\right) \\
\leqslant & \begin{cases}C\left(x_{i}^{1-b}-1\right) x^{b-1-m} & \text { for } 0<x<x_{i}, \\
C x^{b-1-m} & \text { for } x_{i}<x \leqslant 1 \text { if } b<3, \\
C x^{b-1-m} \log (2 / x) & \text { for } x_{i}<x \leqslant 1 \text { if } b=3, \\
C x^{2-m} & \text { for } x_{i}<x \leqslant 1 \text { if } b>3,\end{cases}
\end{aligned}
$$


where in the last step we have used the fact that

$$
|g(x)| \leqslant C x^{b-1} x_{i}^{1-b} \text { for } x<x_{i}
$$

this follows easily from (4.7) by the regularizing effect of $K$, once we know that $g$ is bounded.

Now let $x_{m}$ be a nodal point such that $h / 2<x_{m} \leqslant 3 h / 2$, and note that we may as well assume that $x_{i} \geqslant x_{m}$, in view of Theorem 3. Using Lemma 2, we then have

$$
\begin{aligned}
& \sum_{j \leqslant m}\left(\left\|x(g-\tilde{g})^{\prime}\right\|_{1, I_{j}}+\|g-\tilde{g}\|_{1, I_{j}}\right) \\
& \quad \leqslant C h \sum_{j \leqslant m}\left(\left\|x g^{\prime \prime}\right\|_{1, I_{j}}+\left\|g^{\prime}\right\|_{1, I_{j}}\right) \\
& \quad \leqslant C h \begin{cases}\log \left(1 / x_{i}\right) \int_{0}^{x_{m}} y d y \leqslant C \log \left(1 / x_{i}\right) h^{3} & \text { for } b=1, \\
x_{i}^{1-b} \int_{0}^{x_{m}} y^{b-2} d y \leqslant C x_{i}^{1-b} h^{b} & \text { for } b>1 .\end{cases}
\end{aligned}
$$

Similarly, we have for $b=1$,

$$
\begin{aligned}
& \sum_{j>m}\left(\left\|x(g-\tilde{g})^{\prime}\right\|_{1, I_{j}}+\|g-\tilde{g}\|_{1, I_{j}}\right) \\
& \leqslant C h^{r-1} \sum_{j>m}\left(\left\|x g^{(r)}\right\|_{1, I_{j}}+\left\|g^{(r-1)}\right\|_{1, I_{j}}\right) \\
& \leqslant C h^{r-1}\left(\log \frac{2}{x_{i}} \int_{x_{m}}^{x_{i}} y^{3-r} d y+\int_{x_{i}}^{1} y^{1-r} d y\right) \\
& \leqslant C \begin{cases}h^{2} x_{i}^{-1} & \text { for } r=3, \\
h^{3}\left(\log (1 / h) \log \left(1 / x_{i}\right)+x_{i}^{-2}\right) & \text { for } r=4, \\
\left(h^{3} \log \left(1 / x_{i}\right)+h^{r-1} x_{i}^{2-r}\right) & \text { for } r>4,\end{cases}
\end{aligned}
$$

whereas for $b>1$ we have

$$
\begin{aligned}
& \sum_{j>m}\left(\left\|x(g-\tilde{g})^{\prime}\right\|_{1, I_{j}}+\|g-\tilde{g}\|_{1, I_{l}}\right) \\
& \leqslant C h^{r-1}\left(x_{i}^{1-b} \int_{x_{m}}^{x_{i}} y^{b-r} d y+\int_{x_{i}}^{1} y^{\min (b-r .3-r)}(\log (1 / y))^{\bar{b}} d y\right) \\
& \leqslant C \begin{cases}h^{r-1} x_{i}^{2-r} & \text { for } r<b+1, \\
h^{r-1} \log (1 / h) x_{i}^{2-r} & \text { for } r=b+1, \\
h^{b} x_{i}^{1-b} & \text { for } r>b+1,\end{cases}
\end{aligned}
$$

where $\bar{b}=1$ if $b=3, \bar{b}=0$ otherwise. Together, our estimates now prove Theorem 4.

We shall now consider meshes which are refined near $x=0$ in such a way that

$$
\mu h x_{i}^{\alpha} \leqslant h_{i} \leqslant h x_{i}^{\alpha} \text { for } i=1, \ldots, N,
$$

where $\mu$ is a positive constant, and $\alpha \in[0,1)$ is a parameter. Note that the number of mesh points in such a partition is of the same order $O(1 / h)$, if $\alpha<1$, as for a quasi-uniform mesh which corresponds to the case $\alpha=0$. 
We can now prove the following

THEOREM 5. Let $f$ and $u$ be as in Theorem 4, and assume that we are given a family of refined meshes as above and the corresponding spaces $S_{h}$ such that $(r-2) /(2 r-2)$ $<\alpha<1$ if $b=1,(r-2) /(2 r-2) \leqslant \alpha<1$ if $b>1$, and let $u_{h} \in S_{h}$ be the solution of (1.3). Then, for $h$ sufficiently small we have

$$
\max _{1 \leqslant i \leqslant N}\left|e\left(x_{i}\right)\right| \leqslant C h^{2 r-2}\left\|u^{(r)}\right\|_{\infty}
$$

where $C$ only depends on $b, \lambda / \lambda_{1},\left\|f_{u}\right\|_{\infty}, \mu, r$, and $\alpha$.

Proof. From (4.6) we have

$$
\begin{aligned}
\left|e\left(x_{i}\right)\right| \leqslant & \left.\sum_{j}\left\|e^{\prime}\right\|_{\infty, I_{j}}\left(\left\|x(g-\tilde{g})^{\prime}\right\|_{1, I_{j}}+(b-1) \| g-\tilde{g}\right) \|_{1, I_{j}}\right) \\
& +C \sum_{j}\|e\|_{\infty, I_{j}}\|x(g-\tilde{g})\|_{1, I_{j}} \\
\leqslant & C \sum_{j}\left\|e^{\prime}\right\|_{\infty, I_{j}}\left(\left\|x(g-\tilde{g})^{\prime}\right\|_{1, I_{j}}+\|g-\tilde{g}\|_{1, I_{j}}\right) \\
& +C \max _{1 \leqslant j \leqslant N}\left|e\left(x_{j}\right)\right| \sum_{j}\|g-\tilde{g}\|_{1, I_{j}},
\end{aligned}
$$

where in the last step we have used the fact that

$$
\|e\|_{\infty, I_{j}} \leqslant\left|e\left(x_{j}\right)\right|+h_{j}\left\|e^{\prime}\right\|_{\infty, I_{j}} .
$$

Let us first estimate the local error $\left\|e^{\prime}\right\|_{\infty, I_{j}}$. With $\rho=u-P_{B} u$ and $\theta=P_{B} u-u_{h}$ as before, and with $\omega_{i}=\left(0, x_{i}\right)$, we have

$$
\left\|e^{\prime}\right\|_{\infty, \omega_{i}} \leqslant\left\|\rho^{\prime}\right\|_{\infty, \omega_{i}}+\left\|\theta^{\prime}\right\|_{\infty, \omega_{i}} .
$$

Using Lemma 3 with $\tilde{u}$ as in Lemma 2, $g=(\tilde{u}-u)^{\prime}$, and $h=-(b-1)(\tilde{u}-u)^{\prime}$, we obtain

$$
\begin{aligned}
\left\|\rho^{\prime}\right\|_{\infty, \omega_{i}} & \leqslant\left\|(u-\tilde{u})^{\prime}\right\|_{\infty, \omega_{i}}+\left\|\left(P_{B}(\tilde{u}-u)\right)^{\prime}\right\|_{\infty, \omega_{i}} \\
& \leqslant C\left\|(u-\tilde{u})^{\prime}\right\|_{\infty, \omega_{i}} \leqslant C h_{i}^{r-1}\left\|u^{(r)}\right\|_{\infty, \omega_{i}},
\end{aligned}
$$

where $C=C(b, r)$. By another application of Lemma 3, now with $g=0$ and $h(\cdot)=p(\cdot) e(\cdot)$, where $p$ is defined as in the proof of Theorem 4, we have

$$
\left\|\theta^{\prime}\right\|_{\infty, \omega_{i}} \leqslant C\|e\|_{\infty, \omega_{i}} \leqslant C\left(\max _{1 \leqslant j \leqslant N}\left|e\left(x_{j}\right)\right|+h_{i}\left\|e^{\prime}\right\|_{\infty, \omega_{i}}\right),
$$

where $C=C(b, f, r)$. We conclude that if $h=\max _{i} h_{i}$ is small enough, then

$$
\left\|e^{\prime}\right\|_{\infty, I_{i}} \leqslant\left\|e^{\prime}\right\|_{\infty, \omega_{i}} \leqslant C h_{i}^{r-1}\left\|u^{(r)}\right\|_{\infty, \omega_{i}}+C \max _{1 \leqslant j \leqslant N}\left|e\left(x_{j}\right)\right|
$$


Let us now estimate the interpolation error $g-\tilde{g}$ in the norms appearing in the first sum in (5.2). On $I_{1}$ we have, using Lemma 2 with $m=1$ and then (4.5),

$$
\left\|x(g-\tilde{g})^{\prime}\right\|_{1, I_{1}}+\|g-\tilde{g}\|_{1, I_{1}} \leqslant C h_{1}\left(\left\|x g^{\prime \prime}\right\|_{1, I_{1}}+\left\|g^{\prime}\right\|_{1, I_{1}}\right) \leqslant C h_{1} .
$$

On the remaining subintervals $I_{i}$ we similarly use Lemma 2 with $m=r-1$ to obtain

$$
\left\|x(g-\tilde{g})^{\prime}\right\|_{1, I_{i}}+\|g-\tilde{g}\|_{1, I_{i}} \leqslant C h_{i}^{r-1}\left(\left\|x g^{(r)}\right\|_{1, I_{i}}+\left\|g^{(r-1)}\right\|_{1, I_{i}}\right) .
$$

Using (5.3), (5.4), and (5.5), we now have from (5.2),

$$
\begin{aligned}
\left|e\left(x_{i}\right)\right| \leqslant & C\left\|u^{(r)}\right\|_{\infty}\left(h_{1}^{r}+\sum_{j>1} h_{j}^{2(r-1)}\left(\left\|x g^{(r)}\right\|_{1, I_{j}}+\left\|g^{(r-1)}\right\|_{1, I_{j}}\right)\right) \\
& +C \max _{1 \leqslant j \leqslant N} \mid e\left(x_{j}\right) \|\left(h_{1}+\sum_{j>1} h_{j}^{r-1}\left(\left\|x g^{(r)}\right\|_{1, I_{j}}+\left\|g^{(r-1)}\right\|_{1, I_{j}}\right)\right) \\
\leqslant & C\left\|u^{(r)}\right\|_{\infty}\left(h^{r /(1-\alpha)}+h^{2 r-2} \sum_{j>1} x_{j}^{\alpha(2 r-2)}\left(\left\|x g^{(r)}\right\|_{1, I_{j}}+\left\|g^{(r-1)}\right\|_{1, I_{j}}\right)\right) \\
& +\frac{1}{2} \max _{1 \leqslant j \leqslant N}\left|e\left(x_{j}\right)\right|,
\end{aligned}
$$

if $h$ is small enough, since in view of our above estimates for $g^{(r)}(x)$, we find that

$$
\sum_{j>1} h_{j}^{r-1}\left(\left\|x g^{(r)}\right\|_{I_{j}}+\left\|g^{(r-1)}\right\|_{1, I_{j}}\right)
$$

tends to zero with $h$. We thus have

$$
\begin{aligned}
& \max _{1 \leqslant i \leqslant N}\left|e\left(x_{i}\right)\right| \\
& \quad \leqslant C\left\|u^{(r)}\right\|_{\infty}\left(h^{r /(1-\alpha)}+h^{2 r-2} \sum_{j>1} x_{i}^{\alpha(2 r-2)}\left(\left\|x g^{(r)}\right\|_{1, I_{j}}+\left\|g^{(r-1)}\right\|_{1, I_{j}}\right)\right) .
\end{aligned}
$$

To complete the proof, we now note that for $\alpha \geqslant(r-2) /(2 r-2)$ we have that $h^{r /(1-\alpha)} \leqslant h^{2 r-2}$, and for $\alpha>(r-2) /(2 r-2)$ if $b=1, \alpha \geqslant(r-2) /(2 r-2)$ if $b>1$, we have from our estimates for $g$ that

$$
\begin{aligned}
& \sum_{j>1} x_{j}^{\alpha(2 r-2)}\left(\left\|x g^{(r)}\right\|_{1, I_{j}}+\left\|g^{(r-1)}\right\|_{1, I_{j}}\right) \\
& \leqslant\left\{\begin{array}{l}
C\left(\log \left(1 / x_{i}\right) \int_{x_{1}}^{x_{i}} y^{\alpha(2 r-2)+3-r} d y+\int_{x_{i}}^{1} y^{\alpha(2 r-2)+1-r} d y\right) \leqslant C, \\
C\left(x_{i}^{1-b} \int_{x_{1}}^{x_{i}} y^{\alpha(2 r-2)+b-r} d y+\int_{x_{i}}^{1} y^{\alpha(2 r-2)+\min (b-r, 3-r)}\left(\log \frac{1}{y}\right)^{\bar{b}} d y\right) \leqslant C,
\end{array}\right.
\end{aligned}
$$

for $b=1$ and $b>1$, respectively, where $\bar{b}=1$ if $b=3, \bar{b}=0$ otherwise. This completes the proof of Theorem 5.

6. Numerical Tests. Here we present some numerical results from test calculations using our above discretization method (1.3) for the stationary problem (1.1). The 


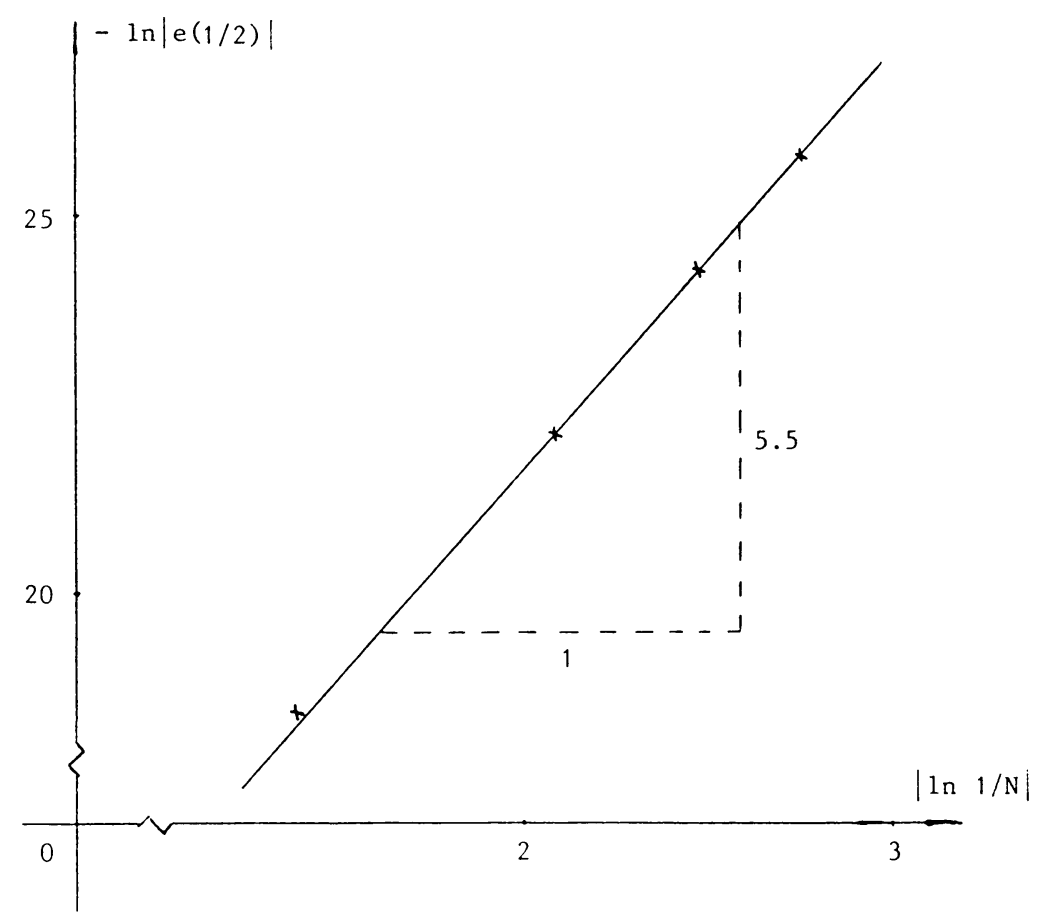

FIGURE 1

results support our theoretical conclusions in Theorems 4 and 5 and indicate that the results obtained there are best possible.

We have considered two cases as follows:

Case 1. We take $b=2.5$ and $f=1-e-x-2.5\left(e^{x}-1\right) / x-u$, which corresponds to an exact solution of (1.1) given by $u=1-e-x+e^{x}$. We compute approximate solutions $u_{h}$ on uniform meshes with $h=1 / N, N=4,8,12,16$ and with $r=4$.

In this case, $r>b+1$, so that according to Theorem 4 we should have

$$
\left|e\left(x_{i}\right)\right| \leqslant C h^{5.5} x_{i}^{-1.5}
$$

In order to check the rate of convergence in terms of $h$, we fix $x_{i}=\frac{1}{2}$ and plot in a $\log$-log diagram (Figure 1) the error $\left|e\left(x_{i}\right)\right|$ as a function of $h=1 / N$ for $N=$ $4,8,12,16$. We find that the computed rate of convergence agrees quite well with 5.5 , which is predicted by Theorem 4 .

We then fix $h=\frac{1}{16}$ and plot the error $\left|e\left(x_{i}\right)\right|$ as a function of $x_{i}$ in another log-log diagram (Figure 2). We then find a relation between $\left|e\left(x_{i}\right)\right|$ and $x_{i}$ which again is in good agreement with the theory, and together our results indicate that the error bound given in Theorem 4 is sharp. 


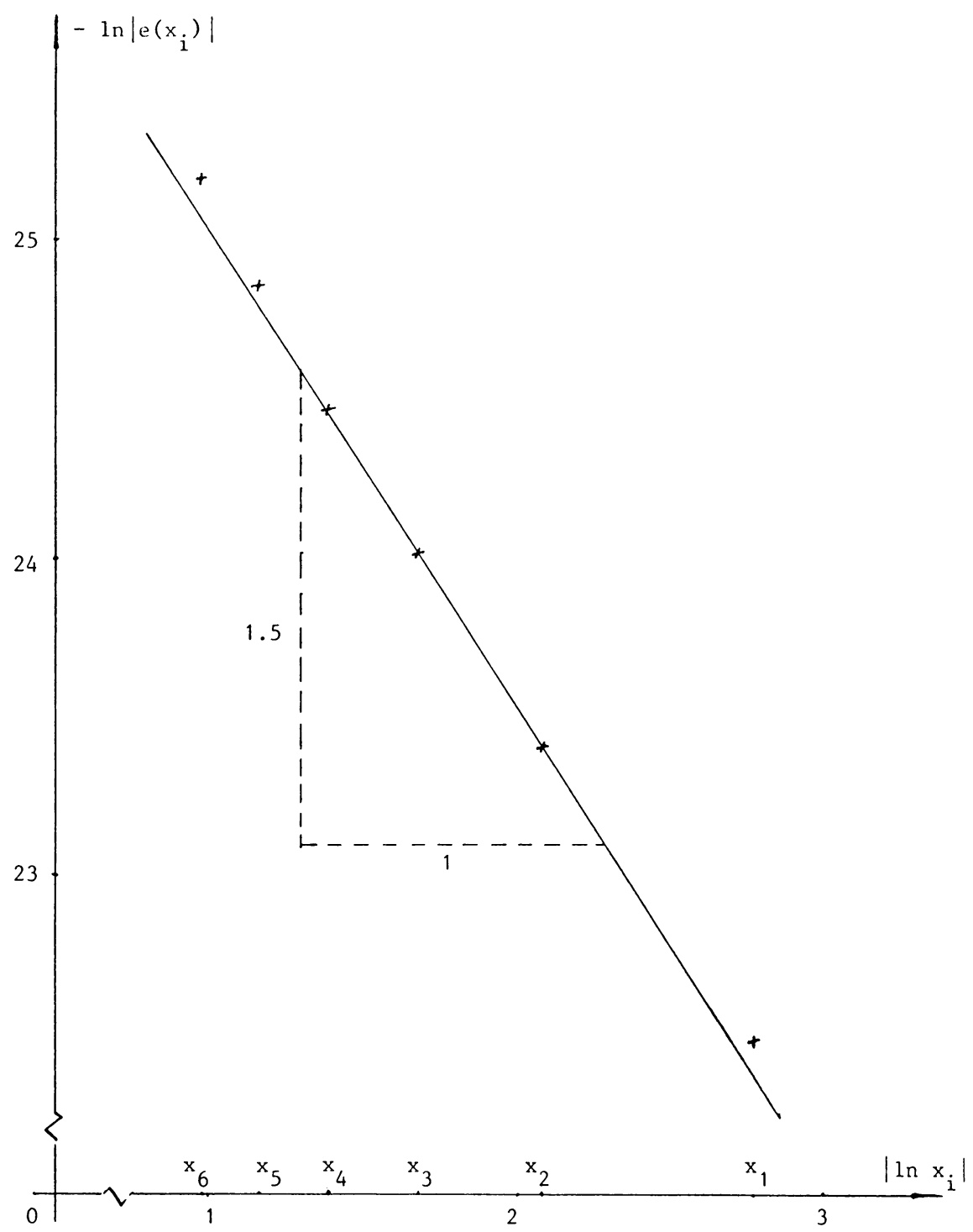

FIGURE 2

Case 2. Here we take $b=1.5$ and $f=1-e-x-1.5\left(e^{x}-1\right) / x-u$, which corresponds to the same exact solution $u$ as before, but now we consider nonuniform meshes with $r=3, h_{i}=h x_{i}^{1 / 4}, h=1 / N, N=4,8,12,16$ as suggested in Theorem 5 , and then expect to have

$$
\max _{i}\left|e\left(x_{i}\right)\right| \leqslant C h^{4}
$$

To check this, we now plot $\max _{i}\left|e\left(x_{i}\right)\right|$ as a function of $h=1 / N$ and find in Figure 3 a computed rate of convergence which again agrees with our theoretical result. 


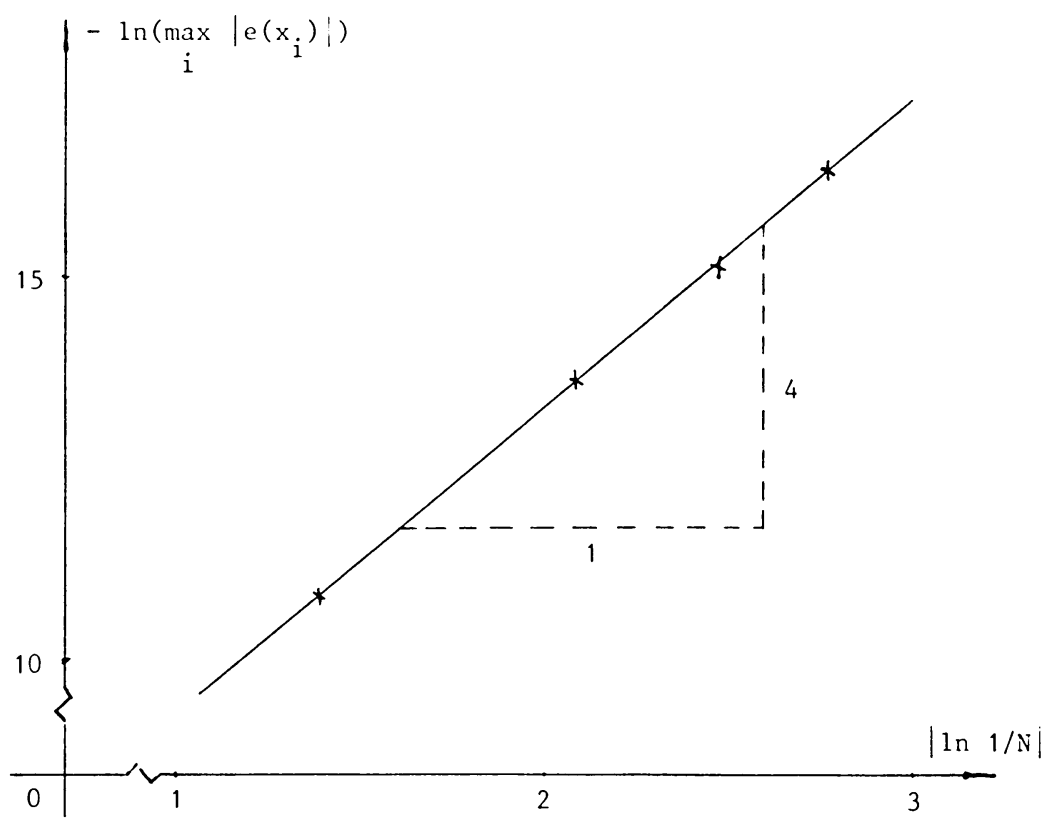

FIGURE 3

Department of Mathematics

Chalmers University of Technology and

The University of Göteborg

S-412 96 Göteborg, Sweden

1. K. ERIKSSON \& V. THOMEE, "Galerkin methods for singular boundary value problems in one space dimension," Math. Comp., v. 42, 1984, pp. 345-367.

2. B. Gidas, Wei Ming Ni \& L. Nirenberg, "Symmetry of positive solutions of nonlinear elliptic equations in $R^{n}$," J. Math. Anal. Appl. Part A, pp. 369-402, Adv. in Math. Suppl. Stud., 7a, Academic Press, New York, 1981.

3. D. JeSPERSEN, "Ritz-Galerkin methods for singular boundary value problems," SIAM J. Numer. Anal., v. 15, 1978, pp. 813-834. 